\title{
CONDITIONAL MEAN DIMENSION
}

\author{
BINGBING LIANG
}

\begin{abstract}
We introduce some notions of conditional mean dimension for a factor map between two topological dynamical systems and discuss their properties. With the help of these notions, we obtain an inequality to estimate the mean dimension of an extension system. The conditional mean dimension for $G$-extensions are computed. We also exhibit some applications in the dynamical embedding problems.
\end{abstract}

\section{INTRODUCTION}

Let $\Gamma$ be a countable amenable group. By a dynamical system $\Gamma \curvearrowright X$, we mean a compact metrizable space $X$ associated with a continuous action of $\Gamma$. Suppose $\Gamma \curvearrowright Y$ is another dynamical system and $\pi: X \rightarrow Y$ is a continuous $\Gamma$-equivariant surjective map, i.e. a factor map between $X$ and $Y$. In such a setting, we call $\Gamma \curvearrowright X$ an extension system and $\Gamma \curvearrowright Y$ a factor system.

Mean (topological) dimension is a newly-introduced dynamical invariant by Gromov [7], which measures the average dimension information of dynamical systems based on the covering dimension for compact Hausdorff spaces. It plays a crucial role in the embedding problem of dynamical systems [8, 9, 10, 11, 12, 19].

Since each fiber $\pi^{-1}(y)$ is a closed subset of the ambient system $\Gamma \curvearrowright X$, taking advantage of the ambient action, we can also discuss the mean dimension $\operatorname{mdim}\left(\pi^{-1}(y), \Gamma\right)$ for the fiber $\pi^{-1}(y)$ (see Definition (2.14). When computing the mean dimension of moduli spaces of Brody curves, Tsukamoto established an inequality to estimate the mean dimension of the extension system in terms of the mean dimension of the factor system [25, Theorem 4.6]. Based on this inequality, he asked the following question [25, Problem 4.8]:

Question 1.1. For a factor map $\pi: X \rightarrow Y$, is it true that $\operatorname{mdim}(X) \leq$ $\operatorname{mdim}(Y)+\sup _{y \in Y} \operatorname{mdim}\left(\pi^{-1}(y), \Gamma\right)$ ?

Date: June, 2021.

2020 Mathematics Subject Classification. Primary 37B02, 54E45.

Key words and phrases. amenable group, conditional mean dimension, Gextension, dynamical embedding. 
Observe that as $\Gamma$ is trivial, the above inequality recovers as the classical Hurewicz's formula [14, Theorem VI 7][6. Theorem 1.12.4, 3.3.10]:

$$
\operatorname{dim}(X) \leq \operatorname{dim}(Y)+\sup _{y \in Y} \operatorname{dim}\left(\pi^{-1}(y)\right)
$$

So Question 1.1 can be regarded as a dynamical concern of the Hurewicz's inequality.

We can also consider Question 1.1 in parallel with entropy theory. Historically, for $\Gamma=\mathbb{Z}$, in [3], Bowen estimated the topological entropy $h(X)$ of $\Gamma \curvearrowright X$ in terms of the entropy of fibers $h\left(\pi^{-1}(y), \Gamma\right)$ for $y \in Y$, i.e.

$$
h(X) \leq h(Y)+\sup _{y \in Y} h\left(\pi^{-1}(y), \Gamma\right) .
$$

In particular, this verifies a conjecture of [1, Conjecture 5] concerning the entropy of a skew product system. Later, some versions of conditional entropy $h(X \mid Y)$ relative to a factor $\Gamma \curvearrowright Y$ were introduced and the related variational principles were established [20, 5, 27]. In particular, it is shown that

$$
h(X \mid Y)=\sup _{y \in Y} h\left(\pi^{-1}(y), \Gamma\right)
$$

in the case $\Gamma=\mathbb{Z}[5]$ and in the general case $\Gamma$ is amenable [27]. This brings us the third motivation to study Question 1.1.

Motivated from these points of view, we introduce some conditional versions of mean dimension relative to a factor system and study their properties. When the factor is trivial, these conditional mean dimensions recover as the mean dimension.

In Section 2, we first define the conditional mean (topological) dimension. We study a class of extensions, called G-extensions, which generalize (topological) principal group extensions (Definition 2.6). A $G$-extension is based on another dynamical system $\Gamma \curvearrowright G$ (typically $G$ is a compact metrizable group and the action is by automorphisms). It turns out the notion of conditional mean dimension brings us a proper notion to strengthen some results on embedding problems of dynamical systems in terms of Rokhlin dimension of the factor systems. We present such applications in Theorem 2.11 and Corollary 2.13. Under certain conditions, Question 1.1 is confirmed.

In Section 3, in terms of notion of conditional mean dimension, we prove an inequality to estimate the mean dimension of an extension system.

Theorem 1.2. For any factor map $\pi: X \rightarrow Y$, we have

$$
\operatorname{mdim}(X) \leq \operatorname{mdim}(Y)+\operatorname{mdim}(X \mid Y) .
$$


The key technique of the proof is to approximate sufficiently large Følners set by smaller Følner sets as in the proof of [25, Theorem 4.6]. In fact, we can slightly adjust the proof to obtain a more general inequality for a composition of two factor maps, or even the setting of the inverse limit of factor maps.

We remind the reader that $\operatorname{mim}(X \mid Y)$ is an upper bound of $\sup _{y \in Y} \operatorname{mdim}\left(\pi^{-1}(y), \Gamma\right)$ (see Proposition 2.16) and in some case they coincide with each other. Thus the above estimation can be thought as a weak version of the inequality in Question 1.1( see Proposition 2.17).

Note that as $X$ is a product system $Y \times Z$ for some dynamical system $\Gamma \curvearrowright Z$ associated with the diagonal action, $\pi$ is the projection map, then we have $\operatorname{mdim}(X \mid Y)=\operatorname{mdim}(Z)$ (see Proposition 2.4). This recovers as the subadditivity formula for Cartesian products of dynamical systems [19, Proposition 2.8].

As a cousin of mean dimension, Lindenstrauss and Weiss introduced the metric mean dimension as an upper bound of mean dimension [19]. This notion is a dynamical analogue of lower box dimension. In Section 4 , we define the conditional version of the metric mean dimension. It is natural to ask whether the conditional metric mean dimension is an upper bound of conditional mean dimension (Question 4.4).

Downarowicz and Serafin introduced the topological fiber entropy given a measure on the factor system [5, Definition 8]. Motivated by this approach, we introduce an analogue for mean dimension. It turns that this mean dimension given a measure serves as a lower bound for the conditional mean dimension (See Propositions 5.4 and 5.5).

Acknowledgements. I am grateful to the inspiring discussion with Hanfeng $\mathrm{Li}$ and the referee's valuable comment. The author is supported by the Research Support Funding GJ10700120 at Soochow University and the Institute of Mathematics of the Polish Academy of Sciences.

\section{Conditional mean topological Dimension}

In this section, we define the notion of conditional mean topological dimension, discuss its properties, and compute some examples.

Let us first recall some machinery of amenable groups in the preparation of defining dynamical invariants. For a countable group $\Gamma$ denote by $\mathcal{F}(\Gamma)$ the set of all nonempty finite subsets of $\Gamma$.

2.1. Amenable groups. For each $K \in \mathcal{F}(\Gamma)$ and $\delta>0$, denote by $\mathcal{B}(K, \delta)$ the set of all $F \in \mathcal{F}(\Gamma)$ satisfying $|\{t \in F: K t \subseteq F\}| \geq$ $(1-\delta)|F| . \Gamma$ is called amenable if $\mathcal{B}(K, \delta)$ is not empty for each pair $(K, \delta)$. 
The collection of pairs $(K, \delta)$ forms a net $\Lambda$ in the sense that $\left(K^{\prime}, \delta^{\prime}\right) \succeq$ $(K, \delta)$ if $K^{\prime} \supseteq K$ and $\delta^{\prime} \leq \delta$. For a real-valued function $\varphi$ defined on $\mathcal{F}(\Gamma) \cup\{\emptyset\}$, we say that $\varphi(F)$ converges to $c \in \mathbb{R}$ when $F \in \mathcal{F}(\Gamma)$ becomes more and more invariant, denoted by $\lim _{F} \varphi(F)=c$, if for any $\varepsilon>0$ there is some $(K, \delta) \in \Lambda$ such that $|\varphi(F)-c|<\varepsilon$ for all $F \in \mathcal{B}(K, \delta)$. In general, $\varlimsup_{F} \varphi(F)$ is defined as

$$
\varlimsup_{F} \varphi(F):=\lim _{(K, \delta) \in \Lambda} \sup _{F \in \mathcal{B}(K, \delta)} \varphi(F) .
$$

In the rest of this paper, $\Gamma$ will always denote a countable amenable group. The following fundamental lemma, due to Ornstein and Weiss, is crucial to define dynamical invariants for amenable group actions [19, Theorem 6.1].

Lemma 2.1. Let $\varphi: \mathcal{F}(\Gamma) \rightarrow[0,+\infty)$ be a map satisfying

(1) $\varphi(F s)=\varphi(F)$ for all $F \in \mathcal{F}(\Gamma)$ and $s \in \Gamma$;

(2) $\varphi\left(F_{1} \cup F_{2}\right) \leq \varphi\left(F_{1}\right)+\varphi\left(F_{2}\right)$ for all $F_{1}, F_{2} \in \mathcal{F}(\Gamma)$.

Then the $\operatorname{limit} \lim _{F} \varphi(F) /|F|$ exists.

2.2. Conditional mean topological dimension. Let $X$ be a compact metrizable space. For two finite open covers $\mathcal{U}$ and $\mathcal{V}$ of $X$, the joining $\mathcal{U} \vee \mathcal{V}$ is defined as $\mathcal{U} \vee \mathcal{V}=\{U \cap V: U \in \mathcal{U}, V \in V\}$. We say $\mathcal{U}$ refines $\mathcal{V}$, denoted by $\mathcal{U} \succeq \mathcal{V}$, if every element of $\mathcal{U}$ is contained in some element of $\mathcal{V}$. Denote by ord $(\mathcal{U})$ the overlapping number of $\mathcal{U}$, i.e.

$$
\operatorname{ord}(\mathcal{U})=\max _{x \in X} \sum_{U \in \mathcal{U}} 1_{U}(x)-1
$$

Now fix a factor map $\pi: X \rightarrow Y$ and a finite open cover $\mathcal{U}$ of $X$. Consider the number $\mathcal{D}(\mathcal{U} \mid Y):=\min ($ ord $(\mathcal{W}))$ for $\mathcal{W}$ ranging over all finite open covers of $X$ such that $\left\{\pi^{-1}(y)\right\}_{y \in Y} \vee \mathcal{W}$ refines $\mathcal{U}$. We put $\mathcal{D}(\mathcal{U}):=\mathcal{D}(\mathcal{U} \mid Y)$ for $Y$ being a singleton. For any $F \in \mathcal{F}(\Gamma)$, denote by $\mathcal{U}^{F}$ the finite open cover $\vee_{s \in F} s^{-1} \mathcal{U}$. To see the function $\varphi: \mathcal{F}(\Gamma) \cup\{\emptyset\} \rightarrow \mathbb{R}$ sending $F$ to $\mathcal{D}\left(\mathcal{U}^{F} \mid Y\right)$ satisfies the conditions of Lemma [2.1, we have a conditional version of [19, Proposition 2.4] to assist us.

Lemma 2.2. Suppose that $\pi: X \rightarrow Y$ is a continuous map and $\mathcal{U}$ is a finite open cover of $X$. Then $\mathcal{D}(\mathcal{U} \mid Y) \leq k$ if and only if there exists a continuous map $f: X \rightarrow P$ for some polyhedron $P$ with $\operatorname{dim}(P)=k$ such that $\left\{f^{-1}(p) \cap \pi^{-1}(y)\right\}_{(p, y) \in P \times Y}$ refines $\mathcal{U}$. 
Proof. Firstly suppose that we have such a continuous map $f: X \rightarrow P$. Let $\varphi: X \rightarrow P \times Y$ be the map sending $x$ to $(f(x), \pi(x))$. By [19, Proposition 2.4], there exists a finite open cover $\mathcal{V}$ of $P \times Y$ such that $\varphi^{-1}(\mathcal{V})$ refines $\mathcal{U}$. Without loss of generality, we may assume that $\mathcal{V}$ is of a form $\mathcal{W} \times \mathcal{V}_{1}$ for some finite open cover $\mathcal{W}$ of $P$ and finite open cover $\mathcal{V}_{1}$ of $Y$ respectively. Then for every $W \in \mathcal{W}$ and $y \in Y$, we have $y \in V$ for some $V \in \mathcal{V}_{1}$ and $\varphi^{-1}(W \times V) \subseteq U$ for some $U \in \mathcal{U}$. Thus

$$
f^{-1}(W) \cap \pi^{-1}(y) \subseteq f^{-1}(W) \cap \pi^{-1}(V)=\varphi^{-1}(W \times V) \subseteq U .
$$

This concludes that $f^{-1}(\mathcal{W}) \vee\left\{\pi^{-1}(y)\right\}_{y}$ refines $\mathcal{U}$. Choose a finite open cover $\mathcal{V}_{2}$ of $P$ refining $\mathcal{W}$ such that ord $\left(\mathcal{V}_{2}\right) \leq \operatorname{dim}(P)$. Then ord $\left(f^{-1}\left(\mathcal{V}_{2}\right)\right) \leq$ ord $\left(\mathcal{V}_{2}\right) \leq \operatorname{dim} P$ and $f^{-1}\left(\mathcal{V}_{2}\right) \vee\left\{\pi^{-1}(y)\right\}_{y}$ refines $\mathcal{U}$. It follows that $\mathcal{D}(\mathcal{U} \mid Y) \leq \operatorname{dim} P=k$.

Now suppose that $\mathcal{D}(\mathcal{U} \mid Y) \leq k$. By definition, there exists a finite open cover $\mathcal{W}$ of $X$ such that $\left\{\pi^{-1}(y)\right\}_{y} \vee \mathcal{W}$ refines $\mathcal{U}$ and ord $(\mathcal{W}) \leq k$. Let $\left\{g_{W}\right\}_{W \in \mathcal{W}}$ be a partition of unity subordinate to $\mathcal{W}$ and $\Delta_{\mathcal{W}}$ be the polyhedron induced from the nerve complex of $\mathcal{W}$. Define the map $g: X \rightarrow \Delta_{\mathcal{W}}$ sending $x$ to $\sum_{W \in \mathcal{W}} g_{W}(x) e_{W}$, where $e_{W}$ stands for the vertex indexed with $W \in \mathcal{W}$. Then for every $q \in \Delta_{\mathcal{W}}, g^{-1}(q)$ is contained in an element $W$ of $\mathcal{W}$ corresponding to a vertex of least dimensional simplex of $\Delta_{\mathcal{W}}$ containing $q$. So for each $y \in Y, g^{-1}(q) \cap$ $\pi^{-1}(y) \subseteq W \cap \pi^{-1}(y) \subseteq U$ for some $U$ in $\mathcal{U}$. Choose a topological embedding $h: \Delta_{\mathcal{W}} \rightarrow P$ for some polyhedron $P$ with $\operatorname{dim} P=k$. Then the map $f:=h \circ g$ is what we need.

From Lemma 2.2, we see that $\varphi$ is sub-additive and hence $\varphi$ satisfies the conditions of Lemma 2.1. Thus the $\operatorname{limit}_{F} \mathcal{D}\left(\mathcal{U}^{F} \mid Y\right) /|F|$ exists.

Definition 2.3. We define the conditional mean topological dimension of $\Gamma \curvearrowright X$ relative to $\Gamma \curvearrowright Y$ as

$$
\operatorname{mdim}(X \mid Y):=\sup _{\mathcal{U}} \lim _{F} \frac{\mathcal{D}\left(\mathcal{U}^{F} \mid Y\right)}{|F|}
$$

for $\mathcal{U}$ running over all finite open covers of $X$. For simplicity, we may also say $\operatorname{mim}(X \mid Y)$ is the conditional mean dimension of $X$ relative to $Y$.

When $Y$ is a singleton, mdim $(X \mid Y)$ recovers the mean topological dimension of $\Gamma \curvearrowright X$, which we denote by $\operatorname{mim}(X)$ (see [19, Definition 2.6]). Moreover, as $\Gamma=\left\{e_{\Gamma}\right\}$ is the trivial group, $\operatorname{mdim}(X)$ recovers the (covering) dimension of $X$, which we denote by $\operatorname{dim}(X)$.

Proposition 2.4. Let $\Gamma \curvearrowright Y$ and $\Gamma \curvearrowright Z$ be two dynamical systems. Let $\Gamma$ act on $Y \times Z$ diagonally and $\pi: Y \times Z \rightarrow Y$ the projection map. Then $\operatorname{mdim}(Y \times Z \mid Y)=\operatorname{mdim}(Z)$. 
Proof. Fix a finite open cover $\mathcal{U}$ of $Z$. To show $\operatorname{mdim}(Z) \leq \operatorname{mdim}(Y \times$ $Z \mid Y)$, for any $F \in \mathcal{F}(\Gamma)$, it suffices to show $\mathcal{D}\left(\mathcal{U}^{F}\right) \leq \mathcal{D}\left(\mathcal{V}^{F} \mid Y\right)$ for $\mathcal{V}:=\{Y \times U: U \in \mathcal{U}\}$

Suppose that $\mathcal{D}\left(\mathcal{V}^{F} \mid Y\right)=$ ord $(\mathcal{W})$ for some finite open cover $\mathcal{W}$ of $Y \times Z$ such that $\mathcal{W} \vee\left\{\pi^{-1}(y)\right\}_{y \in Y}$ refines $\mathcal{V}^{F}$. Consider the topological embedding $\varphi: Z \rightarrow Y \times Z$ sending $z$ to $\left(y_{0}, z\right)$ for some fixed $y_{0}$ in $Y$. Then for every $W \in \mathcal{W}$, there exists $U \in \mathcal{U}^{F}$ such that $W \cap \pi^{-1}\left(y_{0}\right) \subseteq$ $Y \times U$. It follows that $\varphi^{-1}(W) \subseteq U$ and hence $\varphi^{-1}(\mathcal{W})$ refines $\mathcal{U}^{F}$. Thus

$$
\mathcal{D}\left(\mathcal{U}^{F}\right) \leq \operatorname{ord}\left(\varphi^{-1}(\mathcal{W})\right) \leq \operatorname{ord}(\mathcal{W})=\mathcal{D}\left(\mathcal{V}^{F} \mid Y\right)
$$

To show the other direction, for any finite open covers $\mathcal{U}_{0}$ and $\mathcal{V}_{0}$ of $Y$ and $Z$ respectively, we need only to show $\mathcal{D}\left(\left(\mathcal{U}_{0} \times \mathcal{V}_{0}\right)^{F} \mid Y\right) \leq \mathcal{D}\left(\mathcal{V}_{0}^{F}\right)$ for all $F \in \mathcal{F}(\Gamma)$.

Let $\mathcal{D}\left(\mathcal{V}_{0}^{F}\right)=$ ord $(\mathcal{V})$ for some finite open cover $\mathcal{V}$ of $Z$ refining $\mathcal{V}_{0}^{F}$. Denote by $p_{Z}$ the projection map from $Y \times Z$ onto $Z$. Then for any $y \in Y$ and $V \in \mathcal{V}$, one has $p_{Z}^{-1}(V) \cap \pi^{-1}(y)=\{y\} \times V \subseteq U \times V$ for any $U \in \mathcal{U}_{0}^{F}$ containing $y$. That means $p_{Z}^{-1}(\mathcal{V}) \cap\left\{\pi^{-1}(y)\right\}_{y \in Y}$ refines $\left(\mathcal{U}_{0} \times \mathcal{V}_{0}\right)^{F}$. Thus

$$
\mathcal{D}\left(\left(\mathcal{U}_{0} \times \mathcal{V}_{0}\right)^{F} \mid Y\right) \leq \operatorname{ord}\left(p_{Z}^{-1}(\mathcal{V})\right)=\text { ord }(\mathcal{V})=\mathcal{D}\left(\mathcal{V}_{0}^{F}\right)
$$

Now we introduce a metric approach to the conditional mean dimension in line with [4, Theorem 6.5.4]. Let $\pi: X \rightarrow Y$ be a factor map and $\rho$ a compatible metric on $X$. For any $\varepsilon>0$, denote by $\operatorname{Wdim}_{\varepsilon}(X \mid Y, \rho)$ the minimal dimension of a polyhedron $P$ which admits a continuous map $f: X \rightarrow P$ such that $\operatorname{diam}\left(f^{-1}(p) \cap \pi^{-1}(y), \rho\right)<\varepsilon$ for every $(p, y) \in P \times Y$. We call such a map a $(\rho, \varepsilon)$-embedding relative to $Y$. For every $F \in \mathcal{F}(\Gamma)$, denote by $\rho_{F}$ the metric on $X$ defined as

$$
\rho_{F}(x, y):=\max _{s \in F} \rho(s x, s y) .
$$

Then it is easy to check that the function $\mathcal{F}(\Gamma) \cup\{\emptyset\} \rightarrow \mathbb{R}$ sending $F$ to $\operatorname{Wdim}_{\varepsilon}\left(X \mid Y, \rho_{F}\right)$ satisfies the conditions of Lemma 2.1. Thus the $\operatorname{limit} \lim _{F} \frac{\operatorname{Wdim}_{\varepsilon}\left(X \mid Y, \rho_{F}\right)}{|F|}$ exists.

Proposition 2.5. For a compatible metric $\rho$ on $X$, we have

$$
\operatorname{mdim}(X \mid Y)=\sup _{\varepsilon>0} \lim _{F} \frac{\operatorname{Wdim}_{\varepsilon}\left(X \mid Y, \rho_{F}\right)}{|F|} .
$$

Proof. For the direction " $\leq$ ", fix a finite open cover $\mathcal{U}$ of $X$. Picking a Lebesgue number $\lambda$ of $\mathcal{U}$ with respect to $\rho$, it suffices to show

$$
\mathcal{D}\left(\mathcal{U}^{F} \mid Y\right) \leq \operatorname{Wdim}_{\lambda}\left(X \mid Y, \rho_{F}\right)
$$


for every $F \in \mathcal{F}(\Gamma)$. Let $f: X \rightarrow P$ be a continuous map with $\operatorname{dim}(P)=\mathrm{Wdim}_{\lambda}\left(X \mid Y, \rho_{F}\right)$ such that $\operatorname{diam}\left(f^{-1}(p) \cap \pi^{-1}(y), \rho_{F}\right)<\lambda$ for every $(p, y) \in P \times Y$. By choice of $\lambda$, we have that $\left\{f^{-1}(p) \cap \pi^{-1}(y)\right\}_{(p, y)}$ refines $\mathcal{U}^{F}$. Applying Lemma 2.2 to $\mathcal{U}^{F}$, it follows that $\mathcal{D}\left(\mathcal{U}^{F} \mid Y\right) \leq$ $\operatorname{dim}(P)=\mathrm{Wdim}_{\lambda}\left(X \mid Y, \rho_{F}\right)$.

Now we show the converse direction of the equality. Fix $\varepsilon>0$ and pick a finite open cover $\mathcal{U}$ of $X$ consisting of some open sets of the diameter less than $\varepsilon$ under the metric $\rho$. It reduces to show

$$
\mathrm{Wdim}_{\varepsilon}\left(X \mid Y, \rho_{F}\right) \leq \mathcal{D}\left(\mathcal{U}^{F} \mid Y\right)
$$

for each $F \in \mathcal{F}(\Gamma)$. Applying Lemma 2.2 to $\mathcal{U}^{F}$, we have a continuous map $f: X \rightarrow P$ with $\operatorname{dim}(P)=\mathcal{D}\left(\mathcal{U}^{F} \mid Y\right)$ such that $\left\{f^{-1}(p) \cap\right.$ $\left.\pi^{-1}(y)\right\}_{(p, y)}$ refines $\mathcal{U}^{F}$. By choice of $\mathcal{U}$, we see that $f$ is a $\left(\rho_{F}, \varepsilon\right)$ embedding relative to $Y$. Thus

$$
\operatorname{Wdim}_{\varepsilon}\left(X \mid Y, \rho_{F}\right) \leq \operatorname{dim}(P)=\mathcal{D}\left(\mathcal{U}^{F} \mid Y\right) .
$$

2.3. $G$-extensions. Let us compute the conditional mean dimension of $G$-extensions.

Definition 2.6. [3, Page 411] Let $\pi: X \rightarrow Y$ be a factor map and $\Gamma \curvearrowright G$ be another dynamical system. $X$ is called a $G$-extension of $Y$ if there exists a continuous map $X \times G \rightarrow X$ sending $(x, g)$ to $x g$ such that for any $x \in X, g, g^{\prime} \in G$ and $t \in \Gamma$, we have

(1) $\pi^{-1}(\pi(x))=x G$;

(2) $x g=x g^{\prime}$ exactly when $g=g^{\prime}$;

(3) $t(x g)=(t x)(t g)$.

Note that when $G$ is a group and the action $\Gamma \curvearrowright G$ is trivial, the factor map $\pi$ recovers as a principal group extension.

Example 2.7. Let $\Gamma \curvearrowright Y$ and $\Gamma \curvearrowright G$ be two dynamical systems such that $G$ is a compact group and $\Gamma$ acts on $G$ by continuous automorphisms. A (continuous) cocycle is a continuous map $\sigma: \Gamma \times Y \rightarrow G$ such that

$$
\sigma(s t, y)=\sigma(s, t y) \cdot s(\sigma(t, y))
$$

for every $s, t \in \Gamma$ and $y \in Y$. It induces an action of $\Gamma$ on $Y \times G$ by

$$
s(y, g):=(s y, \sigma(s, y) \cdot(s g))
$$

for all $s \in \Gamma, y \in Y$ and $g \in G$. Then $Y \times G$ is a $G$-extension of $Y$ in light of the map $(Y \times G) \times G \rightarrow Y \times G$ sending $((y, g), h)$ to $(y, g h)$. We denote by $Y \times{ }_{\sigma} G$ the $G$-extension from such a cocycle $\sigma$. 
Another source of $G$-extension arise when the underlying systems have group structure. Recall that a dynamical system $\Gamma \curvearrowright X$ is called an algebraic action if $X$ is a compact metrizable group and the action of $\Gamma$ on $X$ is by continuous automorphisms. Let $\pi: X \rightarrow Y$ be a factor map between algebraic actions such that $\pi$ is a group homomorphism. Put $G=\operatorname{ker}(\pi)$. Then $X$ is a $G$-extension given by sending $(x, g) \in$ $X \times G$ to $x g$.

Proposition 2.8. Let $X$ be a G-extension of $Y$ for some compact metrizable space $G$. Then $\operatorname{mdim}(X \mid Y) \geq \operatorname{mdim}(G)$. If $\pi: X \rightarrow Y$ admits a continuous section $\tau: Y \rightarrow X$ in the sense that $\tau$ is continuous such that $\pi \circ \tau=\operatorname{id}_{Y}$, we have $\operatorname{mdim}(X \mid Y)=\operatorname{mdim}(G)$.

Proof. Fix $F \in \mathcal{F}(\Gamma)$. Let $\rho_{X}, \rho_{G}$ be two compatible metrics on $X$ and $G$ respectively.

Pick a point $x_{0}$ from $X$. By definition of $G$-extension, for any $x \in G$ and $g, g^{\prime} \in G, x g=x g^{\prime}$ exactly when $g=g^{\prime}$. Thus by compactness of $X$ and $G$, for any $\varepsilon>0$ there exists $\delta>0$ satisfying the following property: for any $g, g^{\prime} \in G$ such that $\rho_{X}\left(x g, x g^{\prime}\right)<\delta$ for some $x \in X$, we have

$$
\rho_{G}\left(g, g^{\prime}\right)<\varepsilon .
$$

Let $\psi: X \rightarrow P$ be a $\left(\rho_{X, F}, \delta\right)$-embedding relative to $\pi$. Then for any $g, g^{\prime} \in G$ with $\psi\left(x_{0} g\right)=\psi\left(x_{0} g^{\prime}\right)$, since $\pi\left(x_{0} g\right)=\pi\left(x_{0}\right)=\pi\left(x_{0} g^{\prime}\right)$, we have $\rho_{X, F}\left(x_{0} g, x_{0} g^{\prime}\right)<\delta$. By inequality (11), we obtain $\rho_{G, F}\left(g, g^{\prime}\right)<\varepsilon$. Denote by $\varphi$ the map $G \rightarrow X$ sending $g$ to $x_{0} g$. This concludes that the map $\psi \circ \varphi$ is a $\left(\rho_{G, F}, \varepsilon\right)$-embedding. The desired inequality then follows from a limit argument.

Now we assume that $\pi$ admits a continuous cross section $\tau: Y \rightarrow X$. For any $\varepsilon>0$, there exists $\delta>0$ such that

$$
\rho_{X}\left(x g, x g^{\prime}\right)<\varepsilon
$$

for any $x \in X$ and $g, g^{\prime} \in G$ such that $\rho_{G}\left(g, g^{\prime}\right)<\delta$.

Assume that $\varphi: G \rightarrow Q$ is a $\left(\rho_{G, F}, \delta\right)$-embedding for some polyhedron $Q$. For each $x \in X$, since $\pi(\tau(\pi(x)))=\pi(x)$, we have $x, \tau(\pi(x)) \in$ $\pi^{-1}(\pi(x))=x G$ and hence $x=\tau(\pi(x)) g_{x}$ for a unique $g_{x} \in G$. Now define $\psi: X \rightarrow Q$ by sending $x$ to $\varphi\left(g_{x}\right)$. Then the continuity of $\psi$ is guaranteed by the continuity of $\tau$. For any $x, x^{\prime} \in X$ with the same image under $\pi$ and $\psi$, since $\varphi$ is a $\left(\rho_{G, F}, \delta\right)$-embedding, we have $\rho_{G}\left(s g_{x}, s g_{x^{\prime}}\right)<\delta$ for any $s \in F$. By the design of $\delta$, we have

$$
\begin{aligned}
\rho_{X}\left(s x, s x^{\prime}\right) & =\rho_{X}\left(s\left(\tau(\pi(x)) g_{x}\right), s\left(\tau\left(\pi\left(x^{\prime}\right)\right) g_{x^{\prime}}\right)\right) \\
& \left.=\rho_{X}\left((s(\tau(\pi(x))))\left(s g_{x}\right),(s(\tau(\pi(x))))\left(s g_{x^{\prime}}\right)\right)\right)<\varepsilon .
\end{aligned}
$$


That implies that $\operatorname{Wdim}_{\varepsilon}\left(X \mid Y, \rho_{X, F}\right) \leq \operatorname{dim}(Q)$. The inequality then follows by running some limit argument.

Example 2.9. Let $\mathbb{Z} \Gamma$ be the integral group ring of $\Gamma$ and $f \in \mathbb{Z} \Gamma$ (see [22] for more details about group rings). Consider that $\Gamma$ acts on $(\mathbb{R} / \mathbb{Z})^{\Gamma}$ by left shift. Let $R(f): X:=(\mathbb{R} / \mathbb{Z})^{\Gamma} \rightarrow(\mathbb{R} / \mathbb{Z})^{\Gamma}$ be the group homomorphism sending $x$ to $x f$. Set $G:=\operatorname{ker}(R(f))$. Then the induced factor map $\pi_{f}: X \rightarrow Y:=\operatorname{im}(R(f))$ shows that $X$ is a $G$-extension of $Y$. Suppose that $f u f=f$ for some $u \in \mathbb{Z} \Gamma$. Then $\pi_{f}$ admits a continuous section $Y \rightarrow X$ by sending $y$ to $y u$. From Proposition 2.8, we have $\operatorname{mdim}(X \mid Y)=\operatorname{mdim}(G)$.

2.4. Embedding problem. By a dynamical emmbedding $\varphi: X \rightarrow Y$ between two dynamical systems $\Gamma \curvearrowright X$ and $\Gamma \curvearrowright Y$, we mean $\varphi$ is a continuous injective map such that $\varphi(s x)=s \varphi(x)$ for every $s \in \Gamma$ and $x \in X$. With the help of conditional mean dimension, we can strengthen some results regarding the dynamical embedding problem in terms of Rokhlin dimension [13, Theorem 3.1].

The notion of Rokhlin dimension appears in the classification of $C^{*}$ algebras induced from topological dynamical systems. The definition in the setting of topological dynamical systems is due to Winter, explicitly formulated by Szabo for $\mathbb{Z}^{k}$-actions [23, Definition 2.1], and extended to the action of residually finite groups by Szabó, Wu, and Zacharias [24]. In particular, Rokhlin dimension of infinite finitely generated nilpotent group actions is estimated in [24, Corollary 8.5].

Now we can consider the definition for the action of amenable groups.

Definition 2.10. Let $\Gamma \curvearrowright X$ be a continuous action by a countable amenable group $\Gamma$ on a compact metrizable space $X$. We say $\Gamma \curvearrowright X$ has Rokhlin dimension $d$, denoted as

$$
\operatorname{dim}_{\text {Rok }}(X, \Gamma)=d,
$$

if $d$ is the smallest nonnegative integer such that for every finite subset $K$ of $\Gamma$ and every $\delta>0$, there exists $(d+1)$ subsets $F_{0}, \cdots, F_{d} \in \mathcal{B}(K, \delta)$ and $(d+1)$ open sets $U_{0}, \cdots, U_{d}$ satisfying that:

(i) the subsets $\left\{s \overline{U_{i}}\right\}_{s \in F_{i}}$ are pairwise disjoint for every $i=0, \cdots, d$;

(ii) the union $\cup_{i=0}^{d} \sqcup_{s \in F_{i}} s U_{i}$ covers the whole space $X$.

Observe that this definition allows the distinct towers $F_{i} U_{i}$ 's to overlap and Rokhlin dimension does not increase when passing to the extension systems.

With the help of the notion of conditional mean dimension, we can improve the statement of [13, Theorem 3.1] in the following. The proof of [13, Theorem 3.1] works here by applying Lemma 2.12 as a conditional version of [12, Lemma 2.1]. 
Theorem 2.11. Let $D$ be a nonnegative integer and $L$ a positive integer. Suppose that $\pi: X \rightarrow Y$ is a factor map with $\operatorname{dim}_{\text {Rok }}(Y, \Gamma)=D$ and $\operatorname{mim}(X \mid Y)<L / 2$. Then there exists a dynamical embedding from $X$ to $\left(\left([0,1]^{(D+1) L}\right)^{\Gamma}\right) \times Y$ where the later is endowed with the product action from the shift action on $\left([0,1]^{(D+1) L}\right)^{\Gamma}$ and the action $\Gamma \curvearrowright Y$.

The following lemma is a conditional version of [12, Lemma 2.1], whose proof works here.

Lemma 2.12. Let $\pi: X \rightarrow Y$ be a continuous map between compact metrizable space with a compatible metric $\rho$ on $X$. Suppose that $f: X \rightarrow[0,1]^{L}$ is a continuous map such that $\left\|f(x)-f\left(x^{\prime}\right)\right\|_{\infty}<\delta$ for every $x, x^{\prime} \in X$ with $\rho\left(x, x^{\prime}\right)<\varepsilon$. Assume that $\operatorname{Wdim}_{\varepsilon}(X \mid Y, \rho)<L / 2$. Then there exists a $(\rho, \varepsilon)$-embedding $g: X \rightarrow[0,1]^{L}$ relative to $Y$ satisfying that $\sup _{x \in X}\|f(x)-g(x)\|_{\infty}<\delta$.

Combining Theorem 2.11 with Proposition 2.8, we obtain the following dynamical embedding result for $G$-extensions.

Corollary 2.13. Let $X$ be a $G$-extension of $Y$ for some compact metrizable space $G$. Suppose that the factor map $\pi: X \rightarrow Y$ admits a continuous section. Assume that $\operatorname{mdim}(G)<L / 2$ and $\operatorname{dim}_{\mathrm{Rok}}(Y, \Gamma)=D$ for some positive integer $L$ and nonnegative integer $D$. Then there exists a dynamical embedding from $X$ to $\left([0,1]^{(D+1) L}\right)^{\Gamma} \times Y$.

2.5. Mean dimension of fibers. Given a finite open cover $\mathcal{U}$ of $X$, for any closed subset $K$ of $X$, denote by $\left.\mathcal{U}\right|_{K}$ the finite open cover of $K$ restricted from $\mathcal{U}$, i.e. $\left.\mathcal{U}\right|_{K}=\{U \cap K: U \in \mathcal{U}\}$. Taking advantage of $\Gamma$-invariance of $X$, we can similarly consider the mean dimension of $K$ as [7, Section 1.5] and Tsukamoto [25, Remark 4.7].

Definition 2.14. Fix a Følner sequence $\mathcal{F}:=\left\{F_{n}\right\}_{n \geq 1}$ of $\Gamma$, i.e. for any $s \in \Gamma,\left|s F_{n} \Delta F_{n}\right| /\left|F_{n}\right|$ converges to 0 as $n$ goes to the infinity. We define the mean dimension of $K$ as

$$
\operatorname{mdim}(K, \Gamma):=\sup _{\mathcal{U}} \underline{\lim }_{n \rightarrow \infty} \frac{\mathcal{D}\left(\left.\mathcal{U}^{F_{n}}\right|_{K}\right)}{\left|F_{n}\right|}
$$

where $\mathcal{U}$ ranges over all finite open covers of $X$.

By the same argument of Proposition 2.5, we have

Proposition 2.15. Fix a compatible metric $\rho$ on $X$. We have

$$
\operatorname{mdim}(K, \Gamma)=\sup _{\varepsilon>0} \varliminf_{n \rightarrow \infty} \frac{\operatorname{Wdim}_{\varepsilon}\left(K, \rho_{F_{n}}\right)}{\left|F_{n}\right|} .
$$


In particular, considering the fibers of a factor map $\pi: X \rightarrow Y$, in light of metric approach formulas in Propositions 2.5 and 2.15, we have the following estimation.

Proposition 2.16. For every $y \in Y$, we have $\operatorname{mdim}\left(\pi^{-1}(y), \Gamma\right) \leq$ $\operatorname{mdim}(X \mid Y)$.

By a modified argument of Proposition 2.8, we have

Proposition 2.17. Let $X$ be a $G$-extension of $Y$. Then $\operatorname{mdim}(G)=$ $\operatorname{mdim}\left(\pi^{-1}(y), \Gamma\right)$ for every $y \in Y$.

We have a satisfactory answer to Question 1.1 in the following case.

Corollary 2.18. Let $\pi: X \rightarrow Y$ be a factor map of algebraic actions such that $\pi$ is a group homomorphism. Write $G:=\operatorname{ker}(\pi)$. Then $\operatorname{mdim}(G)=\operatorname{mdim}\left(\pi^{-1}(y), \Gamma\right)$ for every $y \in Y$. In particular, we have

$$
\operatorname{mdim}(X)=\operatorname{mdim}(Y)+\sup _{y \in Y} \operatorname{mdim}\left(\pi^{-1}(y), \Gamma\right) .
$$

Proof. Clearly $X$ is a $G$-extension of $Y$. Thus the first statement is true from Proposition 2.17. By the addition formula for mean dimension of algebraic actions [17, Corollary 6.1], we have

$$
\begin{aligned}
\operatorname{mdim}(X) & =\operatorname{mdim}(Y)+\operatorname{mdim}(G) \\
& =\operatorname{mdim}(Y)+\sup _{y \in Y} \operatorname{mdim}\left(\pi^{-1}(y), \Gamma\right) .
\end{aligned}
$$

\section{Proof of Theorem 1.2}

In this section, we give the proof of Theorem 1.2 .

First, we recall the quasi-tiling lemma of amenable groups as follows [21, Page 24, Theorem 6] [15, Theorem 8.3]. In fact, one can require all quasi-tiles contain the identity element $e_{\Gamma}$ of $\Gamma$. Let $\varepsilon>0$ and $F_{1}, \cdots, F_{m} \in \mathcal{F}(\Gamma)$, we say $\left\{F_{j}\right\}_{j=1}^{m}$ are $\varepsilon$-disjoint if there exists $F_{j}^{\prime} \subseteq F_{j}$ for every $j=1, \cdots, m$ such that $\left\{F_{j}\right\}_{j=1}^{m}$ are pairwise disjoint and $\left|F_{j}^{\prime}\right| \geq(1-\varepsilon)\left|F_{j}\right|$ for every $j=1, \cdots, m$.

Lemma 3.1. Let $\varepsilon>0$ and $K \in \mathcal{F}(\Gamma)$. Then there exists $\delta>0$ and $K^{\prime}, F_{1}, \cdots, F_{m} \in \mathcal{F}(\Gamma)$ such that

(1) $e_{\Gamma} \in F_{j} \in \mathcal{B}(K, \varepsilon)$, for all $j=1, \cdots, m$;

(2) For each $A \in \mathcal{B}\left(K^{\prime}, \delta\right)$, there exist $D_{1}, \cdots, D_{m} \in \mathcal{F}(\Gamma)$ such that the family $\left\{F_{j} c: c \in D_{j}, j=1, \cdots, m\right\}$ are $\varepsilon$-disjoint subsets of $A$, and $\left|A \backslash \bigcup_{j=1}^{m} F_{j} D_{j}\right| \leq \varepsilon|A|$.

We call those $F_{j}$ 's quasitiles of $\Gamma$ and $D_{j}$ 's the tiling centers of $A$. 
Proof of Theorem 1.2. Fix a finite open cover $\mathcal{U}$ of $X$. Let $0<\varepsilon<1$ and $K \in \mathcal{F}(\Gamma)$. By Lemma 3.1, there exist $\delta>0, K^{\prime} \in \mathcal{F}(\Gamma)$, and tiles $F_{1}, \cdots, F_{m} \in \mathcal{F}(\Gamma)$, such that each $A \in \mathcal{B}\left(K^{\prime}, \delta\right)$ admits tiling centers $D_{1}, \cdots, D_{m} \in \mathcal{F}(\Gamma)$ satisfying the conditions in Lemma 3.1 .

For each $j=1, \cdots, m$ choose a finite open cover $\mathcal{W}_{j}$ of $X$ such that ord $\left(\mathcal{W}_{j}\right)=\mathcal{D}\left(\mathcal{U}^{F_{j}} \mid Y\right)$ and $\mathcal{W}_{j} \vee\left\{\pi^{-1}(y)\right\}_{y \in Y}$ refines $\mathcal{U}^{F_{j}}$. Without loss of generality, we may assume $\mathcal{W}_{j}^{\prime} \vee\left\{\pi^{-1}(y)\right\}_{y}$ still refines $\mathcal{U}^{F_{j}}$ for $\mathcal{W}_{j}^{\prime}:=\{\bar{W}\}_{W \in \mathcal{W}_{j}}$. Then for each $y \in Y$ and $W \in \mathcal{W}_{j}$ there exists $U \in \mathcal{U}^{F_{j}}$ and an open neighborhood $V_{y, W}$ of $y$ such that

$$
\bar{W} \cap \pi^{-1}\left(V_{y, W}\right) \subseteq U .
$$

By compactness there exists a subfamily $\mathcal{V}_{j}$ of $\left\{\cap_{W \in \mathcal{W}_{j}} V_{y, W}: y \in Y\right\}$ such that $\mathcal{V}_{j}$ still makes an open cover of $Y$. Clearly $\mathcal{W}_{j} \vee \pi^{-1}\left(\mathcal{V}_{j}\right)$ refines $\mathcal{U}^{F_{j}}$. Put $\mathcal{V}=\vee_{j=1}^{m} \mathcal{V}_{j}$ (depending only on $\mathcal{U}$ and $K$ ). It follows that $\mathcal{W}_{j} \vee \pi^{-1}(\mathcal{V})$ refines $\mathcal{U}^{F_{j}}$ for every $j=1, \cdots, m$.

Now for $A \in \mathcal{B}\left(K^{\prime}, \delta\right)$, choose a finite open cover $\mathcal{W}_{A}$ of $Y$ such that ord $\left(\mathcal{W}_{A}\right)=\mathcal{D}\left(\mathcal{V}^{A}\right)$ and $\mathcal{W}_{A}$ refines $\mathcal{V}^{A}$. Since $F_{j}$ contains the identity of $\Gamma$, we have $r \mathcal{W}_{A}$ refines $\mathcal{V}$ for every $r \in D_{j}$ and $j=1, \cdots, m$. By construction of $\mathcal{V}$, we have $\mathcal{W}_{j} \vee \pi^{-1}\left(r \mathcal{W}_{A}\right)$ refines $\mathcal{U}^{F_{j}}$. Hence $\left(\bigvee_{j=1}^{m} \vee_{r \in D_{j}} r^{-1} \mathcal{W}_{j}\right) \vee \pi^{-1}\left(\mathcal{W}_{A}\right)=\bigvee_{j=1}^{m} \vee_{r \in D_{j}} r^{-1}\left(\mathcal{W}_{j} \vee \pi^{-1}\left(r \mathcal{W}_{A}\right)\right)$ refines $\mathcal{U}^{\cup_{j} F_{j} D_{j}}$.Thus

$$
\begin{aligned}
\mathcal{D}\left(\mathcal{U}^{A}\right) & \leq \mathcal{D}\left(\mathcal{U}^{\cup_{j} F_{j} D_{j}}\right)+\mathcal{D}\left(\mathcal{U}^{A \backslash \cup_{j} F_{j} D_{j}}\right) \\
& \leq \operatorname{ord}\left(\left(\vee_{j=1}^{m} \vee_{r \in D_{j}} r^{-1} \mathcal{W}_{j}\right) \vee \pi^{-1}\left(\mathcal{W}_{A}\right)\right)+\varepsilon|A| \mathcal{D}(\mathcal{U}) \\
& \leq \mathcal{D}\left(\mathcal{V}^{A}\right)+\sum_{j}\left|D_{j}\right| \mathcal{D}\left(\mathcal{U}^{F_{j}} \mid Y\right)+\varepsilon|A| \mathcal{D}(\mathcal{U}) .
\end{aligned}
$$

Since $\left\{F_{j} c\right\}_{j, c}$ are $\varepsilon$-disjoint subsets of $A$, we have

$$
\sum_{j=1}^{m}\left|F_{j}\right|\left|D_{j}\right| \leq \frac{|A|}{1-\varepsilon}
$$

It follows that

$$
\sum_{j=1}^{m}\left|D_{j}\right| \mathcal{D}\left(\mathcal{U}^{F_{j}} \mid Y\right)=\sum_{j=1}^{m}\left|F_{j}\right|\left|D_{j}\right| \frac{\mathcal{D}\left(\mathcal{U}^{F_{j}} \mid Y\right)}{\left|F_{j}\right|} \leq \frac{|A|}{1-\varepsilon} \sup _{F \in \mathcal{B}(K, \varepsilon)} \frac{\mathcal{D}\left(\mathcal{U}^{F} \mid Y\right)}{|F|}
$$

So

$$
\frac{\mathcal{D}\left(\mathcal{U}^{A}\right)}{|A|} \leq \frac{\mathcal{D}\left(\mathcal{V}^{A}\right)}{|A|}+\frac{1}{1-\varepsilon} \sup _{F \in \mathcal{B}(K, \varepsilon)} \frac{\mathcal{D}\left(\mathcal{U}^{F} \mid Y\right)}{|F|}+\varepsilon \mathcal{D}(\mathcal{U})
$$

Since $A \in \mathcal{B}\left(K^{\prime}, \delta\right)$ is arbitrary, we get

$$
\operatorname{mdim}(\mathcal{U}) \leq \operatorname{mdim}(Y)+\frac{1}{1-\varepsilon} \sup _{F \in \mathcal{B}(K, \varepsilon)} \frac{\mathcal{D}\left(\mathcal{U}^{F} \mid Y\right)}{|F|}+\varepsilon \mathcal{D}(\mathcal{U})
$$


for $\operatorname{mdim}(\mathcal{U}):=\lim _{F} \frac{\mathcal{D}\left(\mathcal{U}^{F}\right)}{|F|}$. Taking the limit for $K$ and $\varepsilon$, we have

$$
\operatorname{mdim}(\mathcal{U}) \leq \operatorname{mdim}(Y)+\operatorname{mdim}(X \mid Y) .
$$

Since $\mathcal{U}$ is arbitrary, this completes the proof.

Remark 3.2. There is a number of reasons why the converse inequality in Theorem 1.2 can fail. It is well known that Cantor set can continuously map onto any compact metrizable space. In particular, for the action of trivial group, we have the converse inequality in Theorem 1.2 fails for such a surjective map. Moreover, Boltyanskiu constructed an example of a compact metrizable space $X$ such that $\operatorname{dim}(X \times X)<2 \operatorname{dim}(X)$ (See [2]). As a consequence, we know that the converse of inequality in Theorem 1.2 can fail even for a projection map.

Corollary 3.3. Let $\Gamma \curvearrowright Y$ be a dynamical system and $\Gamma \curvearrowright G$ an algebraic action. Suppose that $\sigma: \Gamma \times Y \rightarrow G$ is a continuous cocycle. Then for the induced $G$-extension $\Gamma \curvearrowright Y \times_{\sigma} G$, we have

$$
\operatorname{mdim}\left(Y \times_{\sigma} G\right) \leq \operatorname{mdim}(Y)+\sup _{y \in Y} \operatorname{mdim}\left(\pi^{-1}(y), \Gamma\right) .
$$

Proof. Clearly $Y \times{ }_{\sigma} G$ admits a continuous cross section. Thus by Propositions 2.8 and 2.17, we have $\operatorname{mdim}\left(Y \times{ }_{\sigma} G \mid Y\right)=\operatorname{mdim}(G)=$ $\operatorname{mdim}\left(\pi^{-1}(y), \Gamma\right)$ for every $y \in Y$. Applying Theorem 1.2, we have

$$
\begin{aligned}
\operatorname{mdim}\left(Y \times_{\sigma} G\right) & \leq \operatorname{mdim}(Y)+\operatorname{mdim}\left(Y \times_{\sigma} G \mid Y\right) \\
& =\operatorname{mdim}(Y)+\sup _{y \in Y} \operatorname{mdim}\left(\pi^{-1}(y), \Gamma\right) .
\end{aligned}
$$

\section{CONDITIONAL METRIC MEAN DIMENSION}

In contrast with metric mean dimension, it is natural to consider its conditional version. For a metrizable space $X$ with a compatible metric $\rho$ and $\varepsilon>0$, a subset $E \subseteq X$ is called $(\rho, \varepsilon)$-separating if $\rho\left(x, x^{\prime}\right) \geq \varepsilon$ for every distinct $x, x^{\prime} \in E$. Denote by $N_{\varepsilon}(X, \rho)$ the maximal cardinality of $(\rho, \varepsilon)$-separating subsets of $X$.

Definition 4.1. Let $\Gamma \curvearrowright X$ be a dynamical system. Fix a compatible metric $\rho$ on $X$. Set

$$
N_{\varepsilon}(X \mid Y, \rho)=\max _{y \in Y} N_{\varepsilon}\left(\pi^{-1}(y), \rho\right) .
$$


We define the conditional metric mean dimension of $\Gamma \curvearrowright(X, \rho)$ relative to $\Gamma \curvearrowright Y$ as

$$
\operatorname{mdim}_{\mathrm{M}}(X \mid Y, \rho):=\varliminf_{\varepsilon \rightarrow 0} \varlimsup_{F} \frac{\log N_{\varepsilon}\left(X \mid Y, \rho_{F}\right)}{|\log \varepsilon||F|} .
$$

Again, when $Y$ is a singleton, $\operatorname{mim}_{\mathrm{M}}(X \mid Y, \rho)$ recovers as the metric mean dimension of $\Gamma \curvearrowright(X, \rho)$, which we denote by $\operatorname{mdim}_{M}(X, \rho)$ (see [19, Definition 4.1]).

Remark 4.2. Recall that the mesh of a finite open cover $\mathcal{U}$ for $(X, \rho)$ is defined by

$$
\operatorname{mesh}(\mathcal{U}, \rho):=\max _{U \in \mathcal{U}} \operatorname{diam}(U, \rho) .
$$

In terms of this quantity, one can also give an equivalent definition of conditional metric mean dimension by considering the function $\mathcal{F}(\Gamma) \rightarrow$ $\mathbb{R}$ sending $F$ to

$$
\log \max _{y \in Y} \min _{\operatorname{mesh}\left(\mathcal{U}_{y}, \rho_{F}\right)<\varepsilon}\left|\mathcal{U}_{y}\right|
$$

for $\mathcal{U}_{y}$ ranging over all finite open covers of $\pi^{-1}(y)$. It is easy to check that this function satisfies the conditions of Lemma 2.1.

Proposition 4.3. Let $X$ be a G-extension of $Y$ for some compact metrizable space $G$. Suppose that $\rho_{X}$ and $\rho_{G}$ are two compatible metrics on $X$ and $G$ respectively such that

$$
\rho_{X}\left(x g, x g^{\prime}\right)=\rho_{G}\left(g, g^{\prime}\right)
$$

for all $x \in X$ and $g, g^{\prime} \in G$. Then

$$
\operatorname{mdim}_{\mathrm{M}}\left(X \mid Y, \rho_{X}\right)=\operatorname{mdim}_{\mathrm{M}}\left(G, \rho_{G}\right) .
$$

Proof. By definition, a subset $E$ of $\pi^{-1}(y)$ is of a form $x_{0} G_{0}$ for some $x_{0} \in X$ and $G_{0} \subseteq G$. Then $E$ is $\left(\rho_{F}, \varepsilon\right)$-separating subset of $\pi^{-1}(y)$ if and only if $G_{0}$ is a $\left(\rho_{F}, \varepsilon\right)$-separating subset of $G$. By a limit argument, we have $\operatorname{mdim}_{\mathrm{M}}\left(X \mid Y, \rho_{X}\right) \leq \operatorname{mdim}_{M}\left(G, \rho_{G}\right)$.

To see the converse of equality, it suffices to notice that if a subset $G_{0}$ of $G$ is $\left(\rho_{F}, \varepsilon\right)$-separating, then for every $x \in X, x E$ is a $\left(\rho_{F}, \varepsilon\right)$ separating subset of $\pi^{-1}(\pi(x))$.

Question 4.4. For a factor map $\pi: X \rightarrow Y$, is it true that $\operatorname{mdim}(X \mid Y) \leq$ $\operatorname{mdim}_{\mathrm{M}}(X \mid Y, \rho)$ for every compatible metric $\rho$ on $X$ ?

\section{Mean dimension given a measure}

In this section, we define the mean dimension given a measure on the factor system and discuss its properties. We start with a key lemma. 
Lemma 5.1. Suppose that $\varphi: X \rightarrow Y$ is a continuous map between compact metrizable spaces and $\mathcal{U}$ is a finite open cover of $X$. Then the map $Y \rightarrow \mathbb{R}$ sending y to $\mathcal{D}\left(\left.\mathcal{U}\right|_{\varphi^{-1}(y)}\right)$ is upper semicontinuous.

Proof. Fix $y \in Y$ and put $\mathcal{D}\left(\left.\mathcal{U}\right|_{\varphi^{-1}(y)}\right)=d$. By definition, there exists a finite open cover $\mathcal{V}$ of $X$ such that $\left.\left.\mathcal{V}\right|_{\varphi^{-1}(y)} \succ \mathcal{U}\right|_{\varphi^{-1}(y)}$ and $\operatorname{ord}\left(\left.\mathcal{V}\right|_{\varphi^{-1}(y)}\right)=d$. Write $\mathcal{V}$ as $\mathcal{V}=\left\{V_{i}\right\}_{i \in I}$. Then for any $J \subseteq I$ such that $|J|>d+1$, one has

$$
\left(\cap_{j \in J} V_{j}\right) \cap \varphi^{-1}(y)=\emptyset .
$$

Since $X$ is normal, there exists a finite open cover $\mathcal{V}^{\prime}=\left\{V_{i}^{\prime}\right\}_{i \in I}$ such that $\overline{V_{i}^{\prime}} \subseteq V_{i}$ for all $i \in I$ (see [4, Corollary 1.6.4]). In particular, $\varphi^{-1}(y)$ has the empty intersection with $\cap_{j \in J} \overline{V_{j}^{\prime}}$ for all $J \subseteq I$ such that $|J|>d+1$. Thus we conclude that $y$ sits inside the open set

$$
Y \backslash \varphi\left(\cap_{j \in J} \overline{V_{j}^{\prime}}\right)=\left\{z \in Y: \varphi^{-1}(z) \subseteq\left(\cap_{j \in J} \overline{V_{j}^{\prime}}\right)^{c}\right\}
$$

for each $J \subseteq I$ with $|J|>d+1$. That means, as $z$ approaches to $y$, $\varphi^{-1}(z)$ has the empty intersection with $\cap_{j \in J} V_{j}^{\prime}$ for every $J \subseteq I$ with $|J|>d+1$. So by definition, $\mathcal{D}\left(\left.\mathcal{U}\right|_{\varphi^{-1}(z)}\right) \leq \operatorname{ord}\left(\left.\mathcal{V}^{\prime}\right|_{\varphi^{-1}(z)}\right) \leq d$. This finishes the proof.

Based on this lemma, we are safe to define the measure-theoretic conditional mean dimension.

Definition 5.2. Denote by $M_{\Gamma}(Y)$ the collection of $\Gamma$-invariant Borel probability measures on $Y$. For any $\nu \in M_{\Gamma}(Y)$, set

$$
\mathcal{D}(\mathcal{U} \mid \nu):=\int_{Y} \mathcal{D}\left(\left.\mathcal{U}\right|_{\pi^{-1}(y)}\right) d \nu(y) .
$$

Note that $\mathcal{D}\left(\left.\mathcal{U}^{F s}\right|_{\pi^{-1}(y)}\right)=\mathcal{D}\left(\left.\mathcal{U}^{F}\right|_{\pi^{-1}(s y)}\right)$ for any $s \in \Gamma$. It follows that the function $\mathcal{F}(\Gamma) \cup\{\emptyset\} \rightarrow \mathbb{R}$ sending $F$ to $\mathcal{D}\left(\mathcal{U}^{F} \mid \nu\right)$ satisfies the conditions of Lemma 2.1. We define the mean dimension of $\Gamma \curvearrowright X$ given $\nu$ as

$$
\operatorname{mdim}(X \mid \nu):=\sup _{\mathcal{U}} \lim _{F} \frac{\mathcal{D}\left(\mathcal{U}^{F} \mid \nu\right)}{|F|}
$$

for $\mathcal{U}$ ranging over all finite open covers of $X$.

Example 5.3. In the same setting of Proposition 2.4, it is easy to see that $\operatorname{mim}(Y \times Z \mid \nu)=\operatorname{mdim}(Z)$ for any $\nu \in M_{\Gamma}(Y)$.

Following the similar argument as in the proof of [15, Lemma 6.8] by taking liminf instead, we have

Proposition 5.4. Let $\pi: X \rightarrow Y$ be a factor map. Then

$$
\sup _{\nu \in M_{\Gamma}(Y)} \operatorname{mdim}(X \mid \nu) \leq \sup _{y \in Y} \operatorname{mdim}\left(\pi^{-1}(y), \Gamma\right) .
$$


Combining Proposition 2.16 with 5.4 , we see that conditional mean dimension given a measure serves as a lower bound of conditional mean dimension.

Recall that a finite subset $T$ of $\Gamma$ is called a tile if there exists a subset $C$ of $\Gamma$ such that $\{T c\}_{c \in C}$ makes a partition of $\Gamma$. A Følner sequence $F_{n}$ 's is called a tiling Følner sequence if each $F_{n}$ is a tile. It is well known that all elementary amenable groups including abelian groups admit a tiling Følner sequence [26].

Proposition 5.5. When $\Gamma$ is an abelian group, we have

$$
\sup _{\nu \in M_{\Gamma}(Y)} \operatorname{mdim}(X \mid \nu)=\sup _{y \in Y} \operatorname{mdim}\left(\pi^{-1}(y), \Gamma\right),
$$

where $\operatorname{mdim}\left(\pi^{-1}(y), \Gamma\right)$ is defined along a tiling Følner sequence of $\Gamma$.

Proof. From Proposition 5.4, We need only to prove the for every $y \in Y$ there exists a $\mu \in M_{\Gamma}(Y)$ such that $\operatorname{mdim}\left(\pi^{-1}(y), \Gamma\right) \leq \operatorname{mdim}(X \mid \mu)$.

Fix a finite open cover $\mathcal{U}$ of $X$. For every $F \in \mathcal{F}(\Gamma)$ and $z \in Y$, set $f_{F}(z)=\mathcal{D}\left(\left.\mathcal{U}^{F}\right|_{\pi^{-1}(z)}\right)$. By Lemma 5.1, $f_{F}$ is upper semicontinuous. Note that [16, Lemma 3.6] holds when every $f_{F}$ is upper semicontinuous. Pick a cluster point of the measures $\frac{1}{\left|F_{n}\right|} \sum_{s \in F_{n}} \delta_{s y}$ under the weak*-topology, we have $\mu \in M_{\Gamma}(Y)$. Applying [16, Lemma 3.6] to the measures $\nu_{n}=\delta_{y}$, it follows that

$$
\varliminf_{n \rightarrow \infty} \frac{f_{F_{n}}(y)}{\left|F_{n}\right|}=\varliminf_{n \rightarrow \infty} \int_{Y} \frac{f_{F_{n}}}{\left|F_{n}\right|} d \nu_{n} \leq \lim _{n \rightarrow \infty} \int_{Y} \frac{f_{F_{n}}}{\left|F_{n}\right|} d \mu .
$$

Therefore,

$$
\begin{aligned}
& \operatorname{mdim}\left(\pi^{-1}(y), \Gamma\right)=\sup _{\mathcal{U}} \underline{\lim _{n \rightarrow \infty}} \frac{f_{F_{n}}(y)}{\left|F_{n}\right|} \\
& \leq \sup _{\mathcal{U}} \lim _{n \rightarrow \infty} \int_{Y} \frac{f_{F_{n}}}{\left|F_{n}\right|} d \mu=\operatorname{mdim}(X \mid \mu) .
\end{aligned}
$$

Since $y$ is arbitrary, this finishes the proof.

\section{REFERENCES}

[1] R. L. Adler, A. G. Konheim, and M. H. McAndrew. Topological entropy. Trans. Amer. Math. Soc. 114 (1965) no. 2, 309-319.

[2] V. Boltyanskil. An example of a two-dimensional compactum whose topological square is three-dimensional. Amer. Math. Soc. Translation 1951, (1951), no. 48, 3-6.

[3] R. Bowen. Entropy for group endomorphisms and homogeneous spaces. Trans. Amer. Math. Soc. 153 (1971), 401-414. 
[4] M. Coornaert. Topological Dimension and Dynamical Systems. Translated and revised from the 2005 French original. Universitext. Springer, Cham, 2015.

[5] T. Downarowicz and J. Serafin. Fiber entropy and conditional variational principles in compact non-metrizable spaces. Fund. Math. 172 (2002), no. 3, 217-247.

[6] R. Engelking. Theory of dimensions finite and infinite. Sigma Series in Pure Mathematics, 10. Heldermann Verlag, Lemgo, 1995.

[7] M. Gromov. Topological invariants of dynamical systems and spaces of holomorphic maps. I. Math. Phys. Anal. Geom. 2 (1999), no. 4, 323-415.

[8] Y. Gutman. Embedding $\mathbb{Z}^{k}$-actions in cubical shifts and $\mathbb{Z}^{k}$-symbolic extensions. Ergod. Th. Dynam. Sys. 31 (2011), no. 2, 383-403.

[9] Y. Gutman. Mean dimension and Jaworski-type theorems. Proc. Lond. Math. Soc. (3) 111 (2015), no. 4, 831-850.

[10] Y. Gutman. Embedding topological dynamical systems with periodic points in cubical shifts. Ergod. Th. Dynam. Sys. 37 (2017), no. 2, 512538.

[11] Y. Gutman, E. Lindenstrauss, and M. Tsukamoto, Mean dimension of $\mathbb{Z}^{k}$-actions. Geom. Funct. Anal. 26 (2016), no. 3, 778-817.

[12] Y. Gutman and M. Tsukamoto. Mean dimension and a sharp embedding theorem: extensions of aperiodic subshifts. Ergod. Th. Dynam. Sys. 34 (2014), no. 6, 1888-1896.

[13] Y. Gutman, Y. Qiao, and G. Szabó. The embedding problem in topological dynamics and Takens' theorem. Nonlinearity 31 (2018), no. 2, 597-620.

[14] W. Hurewicz and H. Wallman. Dimension Theory. Princeton Mathematical Series, vol. 4. Princeton University Press, Princeton, N. J., 1941.

[15] H. Li. Compact group automorphisms, addition formulas and FugledeKadison determinants. Ann. of Math. (2) 176 (2012), no. 1, 303-347.

[16] B. Liang and K. Yan. Topological pressure for sub-additive potentials of amenable group actions. J. Funct. Anal. 262 (2012), no. 2, 584-601.

[17] H. Li and B. Liang. Mean dimension, mean rank, and von NeumannLück rank. J. Reine Angew. Math. 739 (2018), 207-240.

[18] H. Li and A. Thom. Entropy, determinants, and $L^{2}$-torsion. J. Amer. Math. Soc. 27 (2014), no. 1, 239-292.

[19] E. Lindenstrauss and B. Weiss. Mean topological dimension. Israel J. Math. 115 (2000), 1-24.

[20] M. Misiurewicz. Topological conditional entropy. Studia Math. 55 (1976), no. 2, 175-200.

[21] D. S. Ornstein and B. Weiss. Entropy and isomorphism theorems for actions of amenable groups. J. Analyse Math. 48 (1987), 1-141.

[22] K. Schmidt. Dynamical systems of algebraic origin. Progress in Mathematics, 128. Birkhäuser Verlag, Basel, 1995. 
[23] G. Szabó. The Rokhlin dimension of topological $\mathbb{Z}^{m}$-actions. Proc. Lond. Math. Soc. (3) 110 (2015), no. 3, 673-694.

[24] G. Szabó, J. Wu, and J. Zacharias. Rokhlin dimension for actions of residually finite groups. Ergod. Th. Dynam. Sys. 39 (2019), no. 8, 22482304 .

[25] M. Tsukamoto. Moduli space of Brody curves, energy and mean dimension. Nagoya Math. J. 192 (2008), 27-58.

[26] B. Weiss. Monotileable amenable groups. Topology, ergodic theory, real algebraic geometry, 257-262, Amer. Math. Soc. Transl. Ser. 2, 202, Adv. Math. Sci., 50, Amer. Math. Soc., Providence, RI, 2001.

[27] K. Yan. Conditional entropy and fiber entropy for amenable group actions. J. Differential Equations 259 (2015), no. 7, 3004-3031.

B.L., Department of Mathematical Science, Soochow University, Suzhou 215006, China., The Institute of Mathematics of the Polish Academy of Sciences, ul. Śniadeckich 8, Warsaw 00-656, Poland

Email address: bbliang@suda.edu.cn, bliang@impan.pl 